\title{
Invarianza Factorial del Cuestionario de Autoconcepto Dimensional AUDIM-M en Hombres y Mujeres Universitarios Mexicanos
}

\author{
Héctor Franco Domínguez (MAPE) \\ Humberto Blanco Vega (Dr.) \\ Perla Jannet Jurado García (Dra.) \\ Susana Ivonne Aguirre Vásquez (Dra.) \\ José René Blanco Ornelas (Dr.) \\ Universidad Autónoma de Chihuahua, México
}

Doi:10.19044/esj.2018.v14n29p231 URL:http://dx.doi.org/10.19044/esj.2018.v14n29p231

\begin{abstract}
The present study analyzes the psychometric properties of the Dimensional Self-Concept Questionnaire (AUDIM-M) in university men and women. The total sample was of 730 participants; 365 women and 365 men, with a means age of 20.55 years $(\mathrm{SD}=1.87)$ and 20.78 years $(\mathrm{SD}=1.90)$ respectively. According to the established psychometric requirements, the confirmatory factor analyzes showed that a four-factor structure (personal selfconcept, physical self-concept, social self-concept and academic self-concept) is viable and suitable for both populations (women and men). In addition, the factorial structure, the factorial loads and the intercepts are considered invariant in the two populations; however, there are differences between the populations for the means of the factors physical self-concept and social selfconcept.
\end{abstract}

Keywords: Self-concept, factorial structure, multi-sample confirmatory factor analysis, measurement invariance.

\section{Resumen}

El presente estudio analiza las propiedades psicométricas del Cuestionario de Autoconcepto Dimensional (AUDIM-M) en hombres y mujeres universitarios. La muestra total fue de 730 participantes; 365 mujeres y 365 hombres, con una edad media de 20.55 años $(\mathrm{DE}=1.87)$ y 20.78 años $(\mathrm{DE}=1.90)$ respectivamente. De acuerdo a los requisitos psicométricos establecidos, los análisis factoriales confirmatorios mostraron que una estructura tetrafactorial (autoconcepto personal, autoconcepto físico, 
autoconcepto social y autoconcepto académico) es viable y adecuada para ambas poblaciones (mujeres y hombres). Además, la estructura factorial, las cargas factoriales y los interceptos se consideran invariantes en las dos poblaciones; sin embargo, existen diferencias entre las poblaciones para las medias de los factores autoconcepto físico y autoconcepto social.

Palabras clave: Autoconcepto, estructura factorial, análisis factorial confirmatorio multimuestra, invarianza de medida.

\section{Introducción:}

En los últimos años, considerables investigaciones han informado sobre las influencias del autoconcepto, como un indicador de satisfacción psicológica y bienestar personal, el cual favorece el sentido de la propia identidad y la relación con la aceptación de sí mismo contribuyendo a la salud, el equilibrio psíquico y como un aspecto relevante para obtener el desarrollo personal y social; dando lugar a una correlación positiva significativa entre el autoconcepto y la salud mental (Garaigordobil, Pérez y Mozaz, 2008; Zhu et al., 2016).

El autoconcepto es muy relevante en el complejo proceso del desarrollo psicosocial de los individuos, donde se enmarca la relevancia del mismo para llegar a entender la manera en que los sujetos regulan sus propias conductas en diferentes contextos tales como, familiar, social, académico, físico y emocional (Torres, Pompa, Meza, Ancer y González, 2010).

La formación y desarrollo del autoconcepto es dinámico y cambiante (Zhu et al., 2016); el autoconcepto aumenta su multidimensionalidad con la edad; en edades muy tempranas no existe una diferencia entre el sí mismo y el entorno, por lo que se tiene un autoconcepto global, no diferenciado y específico de cada situación; al ir aumentando la edad se desarrolla un autoconcepto más diferenciado de forma progresiva (Craven y Marsh, 2008).

En la juventud el autoconcepto juega un papel integrador en la identidad personal, volviéndose más complejo a medida en que va aumentando la edad las autopercepciones varían respecto a las áreas más significativas refiriéndose a atributos físicos, de personalidad, pensamientos, sentimientos, intereses y aficiones, ideas, actitudes, valores y aspiraciones que cada uno va construyendo (Pérez, 2014;Pastor 1998; Alcaide ,2009).

Los indicadores de ajuste psicosocial más importantes son el ajuste escolar y la satisfacción con la vida; el término ajuste escolar es tomado para significar la adaptación a las demandas y características del sistema escolar, así como el grado a lo que los adolescentes se sienten comprometidos y aceptados por el entorno escolar. Por su parte, satisfacción con la vida se manifiesta como un juicio positivo, una estimación subjetiva, de un sentimiento de bienestar en relación consigo mismo y con la propia vida independientemente de las 
condiciones objetivas de su situación actual (Lewis, Malone, Huebner y Valois, 2011; Rodríguez-Fernández y Goñi, 2011).

La construcción de dichos atributos será influenciada por múltiples aspectos desde la valoración personal, mensajes del entorno social, escolar y sobre todo de las valoraciones de las personas consideradas importantes. Todo esto proporciona una base de percepciones, organizaciones y experiencias propias que en consecuencia dan lugar a una regulación de la conducta, impactando también en su equilibrio psicológico, motivación y logro académico (Guillén y Ramírez, 2011; Marsh y Martin, 2011).

El autoconcepto es decisivo y central en el desarrollo de la personalidad, tal como lo destacan las principales teorías psicológicas; un autoconcepto positivo es la base del buen funcionamiento personal, social y profesional. De ahí que lograr un autoconcepto positivo sea uno de los objetivos más pretendidos en numerosos programas de intervención psicológica, educativa, clínica, comunitaria, etc.

Desde la multidimensionalidad del autoconcepto existe una clara carencia de modelos que apunten a integrar los diferentes componentes o dimensiones del autoconcepto que puedan proporcionar en conjunto un relato más completo y más preciso de este constructo, de allí que se propongan medidas más sensibles, concretas y específicas en comparación con las medidas globales, que permitan la medición por separado de las áreas de apariencia física, competencia escolar, autoconcepto general, etc.; tal es el caso del Cuestionario de Autoconcepto Dimensional AUDIM el cual resulta ser un cuestionario fiable y breve en la evaluación de todas las dimensiones básicas del autoconcepto (Amezcua y Pichardo, 2000; Fernández-Zabala, Goñi, Rodríguez-Fernández y Goñi, 2015; Goñi, Madariaga, Axpe y Goñi, 2011).

El presente estudio instrumental (Montero y León, 2005) analiza la división factorial propuesta por Aguirre, Blanco, Peinado, Mondaca y Rangel (2017) para el Cuestionario de Autoconcepto Dimensional (AUDIM-M); lo que se justifica por la importancia de comprobar la estructura factorial de un instrumento y la equivalencia psicométrica del mismo en distintos grupos; ya que en el contexto de la comparación intergrupal, es indispensable plantearse la necesidad de llevar a cabo la adaptación de un instrumento de medida psicológica que cumpla con todos los criterios de equivalencia, pero sobre todo plantearse si la misma estructura factorial es aplicable a distintos grupos de sujetos o, de modo más genérico, a distintas poblaciones (Abalo, Lévy, Rial y Varela, 2006; Arbuckle, 2012).

Este trabajo pretende, por una parte, indagar si se replican los resultados psicométricos propuestos por Aguirre et al. (2017) para el Cuestionario de Autoconcepto Dimensional (AUDIM-M) y, por otra, ampliarlos. Para ello, en primer lugar se comprobará el grado de congruencia de la estructura factorial del AUDIM-M obtenida en el presente estudio y la reportada por Aguirre et al. 
(2017). En segundo lugar, se calcula la invarianza factorial entre las muestras del presente estudio.

\section{Metodología \\ Participantes}

Participaron en el estudio 730 sujetos, 365 mujeres y 365 hombres todos estudiantes universitarios de México. Las edades de las mujeres fluctúan entre los 18 y 26 años, con una media de 20.55 y una desviación estándar de 1.87 años; y las de los hombres fluctúan entre los 18 y 26 años, con una media de 20.78 y una desviación estándar de 1.90 años.

\section{Instrumento}

Cuestionario de Autoconcepto (AUDIM-M) de Aguirre et al. (2017), escala tipo Likert de 15 ítems relacionados con la propia persona (Figura 1); donde el encuestado responde, en una escala del 0 a 10 (falso 0, casi siempre falso 1, 2 y 3, a veces verdadero, a veces falso 4, 5 y 6, casi siempre verdadero 7,8 y 9 y verdadero 10) su grado de acuerdo con cada uno de los aspectos propuestos (escogiendo la respuesta que más se ajuste a su persona). Los ítems del cuestionario se agrupan en cuatro factores: autoconcepto personal (6 ítems), autoconcepto físico (4 ítems), autoconcepto social (3 ítems) y autoconcepto académico ( 2 ítems).

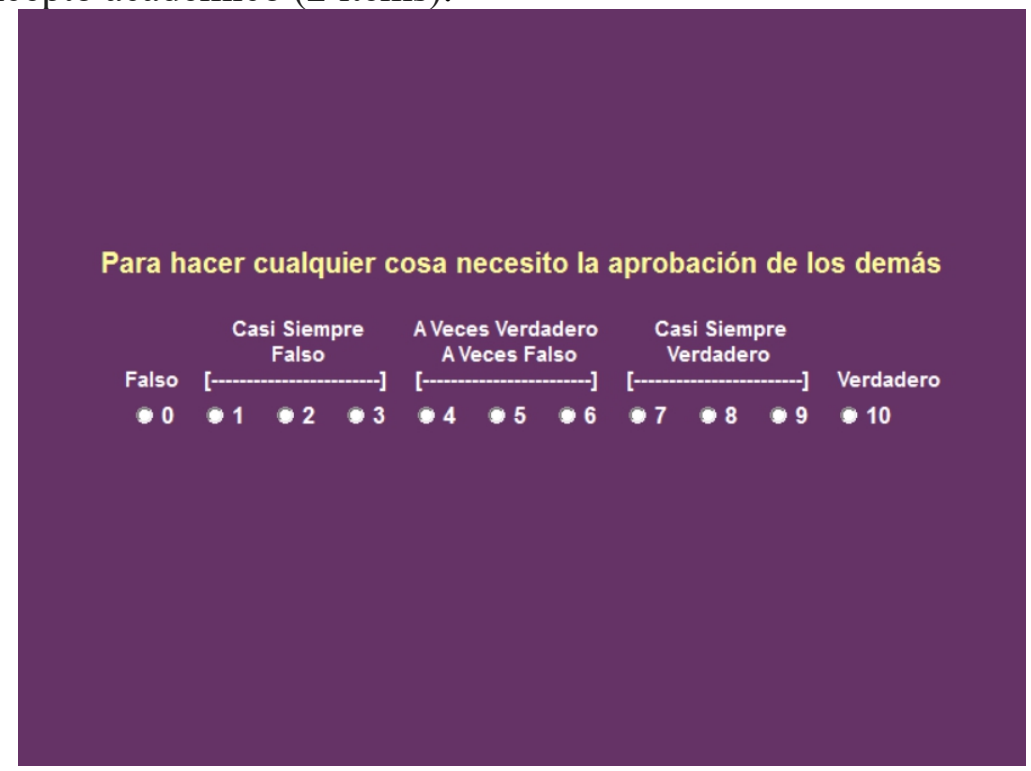

Figura 1. Ejemplo de respuesta para los ítems del cuestionario.

\section{Procedimiento}

Se invitó a participar en el estudio a los alumnos de las licenciaturas que se ofrecen en la Facultad de Ciencias de la Cultura Física (FCCF) de la Universidad Autónoma de Chihuahua. Los que aceptaron participar firmaron 
la carta de aceptación correspondiente. Luego se aplicó el instrumento, antes descrito, por medio de una computadora personal (módulo administrador del instrumento del editor de escalas de ejecución típica), en una sesión de aproximadamente 30 minutos en los laboratorios o centros de cómputo de la FCCF. Al inicio de cada sesión se hizo una pequeña introducción sobre la importancia de la investigación y de cómo acceder al instrumento. Se les solicitó la máxima sinceridad y se les garantizó la confidencialidad de los datos que se obtuvieran. Las instrucciones de cómo responder se encontraban en las primeras pantallas; antes del primer reactivo del instrumento. Al término de la sesión se les agradeció su participación.

Una vez aplicado el instrumento se procedió a recopilar los resultados por medio del módulo generador de resultados del editor de escalas versión 2.0 (Blanco et al., 2013).

Por último los resultados obtenidos, se analizaron mediante el paquete SPSS 18.0 y AMOS 21.0.

\section{Análisis de datos}

El análisis psicométrico se realizó en dos etapas: 1) análisis factorial confirmatorio y 2) análisis de invarianza factorial; con el fin de obtener una prueba que presente las mejores propiedades para la conformación de los puntajes del autoconcepto en universitarios mujeres y hombres.

Para conducir el análisis factorial confirmatorio en cada muestra, se utilizó el software AMOS 21 (Arbuckle, 2012), las varianzas de los términos de error fueron especificados como parámetros libres, en cada variable latente (factor) se fijó uno de los coeficientes estructurales asociados a uno, para que su escala sea igual a la de una de las variables superficiales (ítems). El método de estimación empleado fue el de Máxima Verosimilitud; siguiendo la recomendación de Thompson (2004), en el sentido de que cuando se emplea análisis factorial confirmatorio se debe corroborar no sólo el ajuste de un modelo teórico sino que es recomendable comparar los índices de ajuste de varios modelos alternativos para seleccionar el mejor.

Para evaluar el ajuste del modelo se emplearon el estadístico Chicuadrado, el índice de bondad de ajuste (GFI) y el error cuadrático medio de aproximación (RMSEA) como medidas absolutas de ajuste. El índice de bondad ajustado (AGFI), el Índice Tucker-Lewis (TLI) y el índice de bondad de ajuste comparativo (CFI) como medidas de ajuste incremental. El índice de ajuste normado de parsimonia (PNFI), la razón de Chi-cuadrado sobre los grados de libertad (CMIN/GL) y el Criterio de Información de Akaike (AIC) como medidas de ajuste de parsimonia (Gelabert et al., 2011).

Por último, se llevó a cabo un análisis de la invarianza factorial de los modelos de medida obtenidos, siguiendo las recomendaciones de Abalo et al. 
(2006), y se calculó la fiabilidad de cada una de las dimensiones a través del Alfa de Cronbach y el Coeficiente Omega (Revelle y Zinbarg, 2009).

\section{Resultados}

Con el fin de obtener el cuestionario que presente las mejores propiedades para la conformación de los puntajes del autoconcepto en universitarios mujeres y hombres, se sometieron a prueba dos modelos de medida: el Modelo 1 (M4-15), modelo de cuatro factores acorde a la distribución de los ítems dentro del cuestionario propuesta por Aguirre et al. (2017) y el Modelo 2 (M411), modelo de cuatro factores conforme a los resultados del análisis factorial confirmatorio del modelo M4-15, eliminando cuatro de los ítems que no fueron suficientemente bien explicados por este modelo.

\section{Análisis factoriales confirmatorios modelo M4-15}

De acuerdo a los resultados de la Tabla 1 el análisis factorial confirmatorio de 15 ítems agrupados en cuatro factores (autoconcepto personal, autoconcepto físico, autoconcepto social y autoconcepto académico), modelo M4-15, en la muestra de mujeres es aceptable (GFI .923 y RMSEA .071) y de acuerdo a las medidas de ajuste incremental y de parsimonia significativamente superior al modelo independiente y muy similar al modelo saturado.

Por otro lado, el análisis factorial confirmatorio en la muestra de hombres (Tabla 1), indica que el modelo de medición de cuatro factores (M4-15) es también aceptable (GFI .923 y RMSEA .082) y de acuerdo a las medidas de ajuste incremental y de parsimonia significativamente superior al modelo independiente y muy similar al modelo saturado.

Tabla 1 Índices absolutos, incrementales y de parsimonia para los modelos generados. Análisis factorial confirmatorio para mujeres y hombres modelo M4-15.

\begin{tabular}{|c|c|c|c|c|c|c|c|c|}
\hline \multirow[b]{2}{*}{ Modelo } & \multicolumn{3}{|c|}{ Índices absolutos } & \multicolumn{3}{|c|}{$\begin{array}{c}\text { Índices } \\
\text { incrementales }\end{array}$} & \multicolumn{2}{|c|}{ Índices de parsimonia } \\
\hline & $\chi^{2}$ & GFI & RMSEA & AGFI & TLI & CFI & CMIN/DF & $\mathrm{AIC}$ \\
\hline \multicolumn{9}{|c|}{ Solución factorial para las mujeres } \\
\hline M4-15 & $237.810^{*}$ & .923 & .071 & .890 & .914 & .931 & 2.831 & 309.810 \\
\hline Saturado & 0.000 & 1.000 & & & & 1.000 & & 240.000 \\
\hline Independiente & $2336.530 *$ & .408 & .242 & .323 & .000 & .000 & 22.253 & 2366.530 \\
\hline \multicolumn{9}{|c|}{ Solución factorial para los hombres } \\
\hline M4-15 & $290.911 *$ & .907 & .082 & .868 & .867 & .893 & 3.463 & 362.911 \\
\hline Saturado & 0.000 & 1.000 & & & & 1.000 & & 240.000 \\
\hline Independiente & $2043.732 *$ & .436 & .225 & .355 & .000 & .000 & 19.464 & 2073.732 \\
\hline
\end{tabular}

Nota: ${ }^{*} \mathrm{p}<.05$; GFI = índice de bondad de ajuste; RMSEA = raíz del error medio; AGFI = índice corregido de la bondad de ajuste; TLI = índice de Tucker-Lewis; CFI = índice de ajuste comparativo; $\mathrm{CMIN} / \mathrm{DF}$ = índice de ajuste chi cuadrado dividido por los grados de libertad; $\mathrm{AIC}=$ criterio de información de Akaike 
De acuerdo a los resultados de la Tabla 2, solo dos de los 15 ítems en la muestra de mujeres y cuatro en la de hombres saturan por debajo de .60 en su dimensión prevista. Observándose además, intercorrelaciones de bajas a moderadas entre los factores evidenciando una adecuada validez discriminante entre ellos.

Tabla 2 Soluciones estandarizadas análisis factorial confirmatorio en ambas muestras modelo M4-15.

\begin{tabular}{|c|c|c|c|c|c|c|c|c|}
\hline \multirow[b]{2}{*}{ Ítem } & \multicolumn{4}{|c|}{ Mujeres } & \multicolumn{4}{|c|}{ Hombres } \\
\hline & $\mathrm{F} 1$ & $\mathrm{~F} 2$ & F3 & $\mathrm{F} 4$ & $\mathrm{~F} 1$ & $\mathrm{~F} 2$ & F3 & F4 \\
\hline \multicolumn{9}{|c|}{ Pesos Factoriales } \\
\hline 4 Me siento feliz & .82 & & & & .73 & & & \\
\hline $\begin{array}{l}6 \text { Me siento contento con mi imagen } \\
\text { corporal }\end{array}$ & .68 & & & & .72 & & & \\
\hline $\begin{array}{l}7 \text { Estoy satisfecho con las cosas que } \\
\text { voy consiguiendo en la vida }\end{array}$ & .78 & & & & .85 & & & \\
\hline $11 \mathrm{Me}$ siento una persona afortunada & .78 & & & & .54 & & & \\
\hline 12 Me gusta mi cara & 67 & & & & .60 & & & \\
\hline $\begin{array}{l}14 \text { Estoy orgulloso de cómo voy } \\
\text { dirigiendo mi vida }\end{array}$ & .79 & & & & .76 & & & \\
\hline $\begin{array}{l}1 \text { Puedo correr y hacer ejercicio } \\
\text { durante mucho tiempo sin } \\
\text { cansarme }\end{array}$ & & .74 & & & & .81 & & \\
\hline $\begin{array}{l}9 \text { Tengo más fuerza que la mayoría } \\
\text { de la gente de mi edad }\end{array}$ & & .59 & & & & .50 & & \\
\hline 10 Tengo mucha resistencia física & & .88 & & & & .85 & & \\
\hline 15 Soy fuerte físicamente & & .77 & & & & .66 & & \\
\hline $\begin{array}{l}2 \text { Me considero una persona muy } \\
\text { nerviosa }\end{array}$ & \multicolumn{4}{|c|}{.41} & & \multicolumn{3}{|c|}{. .48} \\
\hline $\begin{array}{l}3 \text { A la hora de tomar una decisión, } \\
\text { dependo demasiado de la opinión } \\
\text { de los demás }\end{array}$ & \multicolumn{4}{|c|}{.81} & & \multicolumn{3}{|c|}{.88} \\
\hline $\begin{array}{l}8 \text { Para hacer cualquier cosa necesito } \\
\text { la aprobación de los demás }\end{array}$ & \multicolumn{4}{|c|}{.78} & & \multicolumn{3}{|c|}{.61} \\
\hline $\begin{array}{l}5 \text { Se me dan bien las asignaturas de } \\
\text { gramática y español }\end{array}$ & & & & .69 & & & & .47 \\
\hline $\begin{array}{l}13 \text { Soy bueno en las asignaturas de } \\
\text { ciencias }\end{array}$ & & & & .69 & & & & .82 \\
\hline \multicolumn{9}{|c|}{ Correlaciones Factoriales } \\
\hline $\mathrm{F} 1$ & - & & & & - & & & \\
\hline $\mathrm{F} 2$ & .24 & - & & & .43 & - & & \\
\hline F3 & .56 & .15 & - & & .47 & .21 & - & \\
\hline $\mathrm{F} 4$ & .36 & .29 & .30 & - & .37 & .40 & .17 & - \\
\hline
\end{tabular}

Nota: $F 1=$ autoconcepto personal, $F 2=$ autoconcepto físico, $F 3=$ autoconcepto social, $F 4$ = autoconcepto académico 


\section{Análisis factoriales confirmatorios modelo M4-11}

De acuerdo a los resultados de la Tabla 3 el análisis factorial confirmatorio de 11 ítems sin los ítems (2, 6, 12 y 15) agrupados en cuatro factores (autoconcepto personal, autoconcepto físico, autoconcepto social y autoconcepto académico), modelo M4-11, en la muestra de mujeres es óptimo (GFI .958 y RMSEA .061) y de acuerdo a las medidas de ajuste incremental y de parsimonia significativamente superior al modelo independiente y muy similar al modelo saturado.

Por otro lado, el análisis factorial confirmatorio en la muestra de hombres (Tabla 3), indica que el modelo de medición de cuatro factores (M4-11) es también aceptable (GFI .923 y RMSEA .082) y de acuerdo a las medidas de ajuste incremental y de parsimonia significativamente superior al modelo independiente y muy similar al modelo saturado.

Tabla 3 Índices absolutos, incrementales y de parsimonia para los modelos generados. Análisis factorial confirmatorio para mujeres y hombres modelo M4-11.

\begin{tabular}{|c|c|c|c|c|c|c|c|c|}
\hline \multirow[b]{2}{*}{ Modelo } & \multicolumn{3}{|c|}{ Índices absolutos } & \multicolumn{3}{|c|}{$\begin{array}{c}\text { Índices } \\
\text { incrementales }\end{array}$} & \multicolumn{2}{|c|}{ Índices de parsimonia } \\
\hline & $\chi^{2}$ & GFI & RMSEA & AGFI & TLI & CFI & CMIN/DF & $\mathrm{AIC}$ \\
\hline \multicolumn{9}{|c|}{ Solución factorial para las mujeres } \\
\hline M4-11 & $88.831^{*}$ & .958 & .061 & .927 & .950 & .966 & 2.338 & 144.831 \\
\hline Saturado & 0.000 & 1.000 & & & & 1.000 & & 132.000 \\
\hline Independiente & $1535.299 *$ & .494 & .272 & .393 & .000 & .000 & 27.915 & 1557.299 \\
\hline \multicolumn{9}{|c|}{ Solución factorial para los hombres } \\
\hline M4-11 & $73.665^{*}$ & .967 & .051 & .943 & .957 & .970 & 1.939 & 129.665 \\
\hline Saturado & 0.000 & 1.000 & & & & 1.000 & & 132.000 \\
\hline Independiente & $1254.516^{*}$ & .549 & .245 & .459 & .000 & .000 & 22.809 & 1276.516 \\
\hline
\end{tabular}

De acuerdo a los resultados de la Tabla 4, solo uno de los 11 ítems en la muestra de mujeres y tres en la de hombres saturan por debajo de .60 en su dimensión prevista. Observándose además, intercorrelaciones de bajas a moderadas entre los factores evidenciando una adecuada validez discriminante entre ellos. 
Tabla 4 Soluciones estandarizadas análisis factorial confirmatorio en ambas muestras modelo M4-11.

\begin{tabular}{|c|c|c|c|c|c|c|c|c|}
\hline \multirow[b]{2}{*}{ Ítem } & \multicolumn{4}{|c|}{ Mujeres } & \multicolumn{4}{|c|}{ Hombres } \\
\hline & F1 & $\mathrm{F} 2$ & F3 & $\mathrm{F} 4$ & $\mathrm{~F} 1$ & $\mathrm{~F} 2$ & F3 & F4 \\
\hline \multicolumn{9}{|c|}{ Pesos Factoriales } \\
\hline 4 Me siento feliz & .82 & & & & .74 & & & \\
\hline $\begin{array}{l}7 \text { Estoy satisfecho con las cosas que } \\
\text { voy consiguiendo en la vida }\end{array}$ & .77 & & & & .86 & & & \\
\hline $11 \mathrm{Me}$ siento una persona afortunada & .78 & & & & .52 & & & \\
\hline $\begin{array}{l}14 \text { Estoy orgulloso de cómo voy } \\
\text { dirigiendo mi vida }\end{array}$ & .81 & & & & .78 & & & \\
\hline $\begin{array}{l}1 \text { Puedo correr y hacer ejercicio } \\
\text { durante mucho tiempo sin cansarme }\end{array}$ & & .73 & & & & .83 & & \\
\hline $\begin{array}{l}9 \text { Tengo más fuerza que la mayoría } \\
\text { de la gente de mi edad }\end{array}$ & & .54 & & & & .43 & & \\
\hline 10 Tengo mucha resistencia física & & .93 & & & & .88 & & \\
\hline $\begin{array}{l}3 \text { A la hora de tomar una decisión, } \\
\text { dependo demasiado de la opinión } \\
\text { de los demás }\end{array}$ & & & .77 & & & & .85 & \\
\hline $\begin{array}{l}8 \text { Para hacer cualquier cosa necesito } \\
\text { la aprobación de los demás }\end{array}$ & & & .83 & & & & .64 & \\
\hline $\begin{array}{l}5 \text { Se me dan bien las asignaturas de } \\
\text { gramática y español }\end{array}$ & & & & .66 & & & & .45 \\
\hline $\begin{array}{l}13 \text { Soy bueno en las asignaturas de } \\
\text { ciencias }\end{array}$ & & & & .72 & & & & .86 \\
\hline \multicolumn{9}{|c|}{ Correlaciones Factoriales } \\
\hline F1 & - & & & & - & & & \\
\hline $\mathrm{F} 2$ & .17 & - & & & .33 & - & & \\
\hline F3 & .55 & .09 & - & & .48 & .14 & - & \\
\hline $\mathrm{F} 4$ & .34 & .23 & .30 & - & .33 & .36 & .17 & - \\
\hline
\end{tabular}

Nota: $F 1=$ autoconcepto personal, $F 2=$ autoconcepto físico, $F 3=$ autoconcepto social, F4 = autoconcepto académico

\section{Invarianza de la estructura factorial entre mujeres y hombres universitarios}

Los índices de ajuste obtenidos (Tabla 5) permiten aceptar la equivalencia de los modelos de medida básicos entre las dos muestras. Aunque el valor de Chi-cuadrado excede al exigido para aceptar la hipótesis de invarianza, los índices $\mathrm{GFI}=.962, \mathrm{CFI}=.968$, RMSEA $=.040$ y $\mathrm{AIC}=274.495$ contradicen esta conclusión lo que nos permite aceptar el modelo base de la invarianza (modelo sin restricciones).

Añadiendo al modelo base restricciones sobre las cargas factoriales caracterizamos la invarianza métrica. Los valores que se recogen en la tabla 5 permiten aceptar este nivel de invarianza. El índice de ajuste general (GFI 
.957) y el error cuadrático medio de aproximación (RMSEA .042) siguen aportando información convergente en esta dirección. Además, el criterio de información de Akaike (AIC 286.704) y el índice comparativo de Bentler (CFI .961) no sufren grandes variaciones respecto al modelo anterior. Haciendo uso del criterio para la evaluación de los modelos anidados propuesto por Cheung y Rensvold (2002), quiénes sugieren que si el cálculo de la diferencia de los CFI de ambos modelos anidados disminuye en .01 o menos, se da por bueno el modelo restringido y por tanto el cumplimiento de la invarianza factorial; la diferencia entre CFIs obtenida permite aceptar el modelo de invarianza métrica. Podemos concluir hasta ahora que las cargas factoriales son equivalentes en las dos muestras.

Una vez demostrada la invarianza métrica entre las muestras, pasamos a evaluar la equivalencia entre interceptos (invarianza factorial fuerte). Los índices (Tabla 5) muestran un ajuste aceptable de este modelo, tanto evaluado de modo independiente como analizándolo respecto a su anidamiento con el modelo de invarianza métrica. La diferencia entre los índices comparativos de Bentler es de cinco milésimas; el índice de ajuste general es .952 y el error cuadrático medio de aproximación es .042. Aceptada la invarianza fuerte, los dos modelos evaluados son equivalentes respecto a los coeficientes factoriales $\mathrm{y}$ a los interceptos.

Tabla 5 Índices de bondad de ajuste de cada uno de los modelos puestos a prueba en la invarianza factorial.

\begin{tabular}{lccccccc}
\hline \multirow{2}{*}{ Modelo } & \multicolumn{7}{c}{ Índice de Ajuste } \\
\cline { 2 - 8 } & $\chi^{2}$ & gl & GFI & PNFI & CFI & RMSEA & AIC \\
\hline Modelo sin restricciones & $162.495^{*}$ & 76 & .962 & .651 & .968 & .040 & 274.495 \\
Invarianza métrica & $188.704^{*}$ & 83 & .957 & .704 & .961 & .042 & 286.704 \\
Invarianza factorial fuerte & $211.150^{*}$ & 93 & .952 & .781 & .956 & .042 & 289.150 \\
\hline
\end{tabular}

Nota: $* \mathrm{p}<.05 ;$ GFI $=$ índice de bondad de ajuste; PNFI = índice de ajuste normado de parsimonia; $\mathrm{CFI}=$ índice de ajuste comparativo; RMSEA = raíz del error medio; AIC = criterio de Información de Akaike

Los factores obtenidos en los análisis factoriales confirmatorios alcanzan, en su mayoría valores de consistencia interna por encima de .70 en ambas muestras (mujeres y hombres); evidenciando una consistencia interna adecuada para este tipo de subescalas, particularmente si se considera el número reducido de ítems (Tabla 6).

Tabla 6. Coeficiente omega y alfa para los factores obtenidos.

\begin{tabular}{lcccc}
\hline \multirow{2}{*}{\multicolumn{1}{c}{ Factor }} & \multicolumn{2}{c}{ Mujeres } & \multicolumn{2}{c}{ Hombres } \\
\cline { 2 - 5 } & $\Omega$ & $\square$ & $\Omega$ & $\square$ \\
\hline autoconcepto personal & .873 & .872 & .821 & .802 \\
autoconcepto físico & .787 & .760 & .772 & .735 \\
autoconcepto social & .781 & .774 & .719 & .701 \\
autoconcepto académico & .645 & .635 & .619 & .550 \\
\hline
\end{tabular}




\section{Contrastes de las medias de los factores entre mujeres y hombres}

Una vez comprobada la invarianza factorial, las diferencias entre las medias de los factores de los dos grupos se estimaron tomando como referente la muestra de hombres, fijando en 0 el valor de las medias para dicha muestra y estimando libremente el valor de las medias para la muestra de mujeres. Las restricciones sobre los coeficientes de regresión e interceptos, requeridos para los contrastes entre las medias se realizaron automáticamente mediante el software AMOS 21 (Arbuckle, 2012). Los resultados de las comparaciones entre medias indicaron que los hombres presentan una mejor percepción de su autoconcepto en los factores "autoconcepto físico" $(-2.213, \mathrm{p}<.001)$, "autoconcepto social" $-0.477, \mathrm{p}<.05)$ y sin diferencias significativas en los factores "autoconcepto personal" y "autoconcepto académico".

\section{Conclusión:}

De los resultados mostrados y de su análisis, tomando en cuenta que el objetivo principal de este estudio fue el de examinar la estructura factorial del cuestionario AUDIM-M y la medición de la invariancia de dicha estructura en alumnas y alumnos universitarios, se pueden obtener las siguientes conclusiones:

1) El Análisis Factorial Confirmatorio, en ambas muestras, indicó que el ajuste de los datos al modelo teórico de 11 ítems agrupados en cuatro factores es óptimo; resultados que en general concuerdan con los reportados por Aguirre et al. (2017). Al mismo tiempo que los cuatro factores así obtenidos presentan en general saturaciones factoriales estandarizadas adecuadas. Por su parte los factores correlacionan entre sí de forma positiva y estadísticamente significativa lo cual muestra que a medida que aumenta la percepción de un mejor autoconcepto en alguno de los factores, también aumenta en los otros.

2) En general los cuatro factores obtenidos muestran una consistencia interna adecuada.

3) Conjuntamente con todo lo antes dicho, los resultados del análisis de la invarianza factorial entre las muestras; indican una alta congruencia entre pares de factores. Lo que sugiere la existencia de fuertes evidencias de la validación cruzada de la medida y por tanto de la estabilidad de la estructura, hasta que no se demuestre lo contrario.

4) Las comparaciones entre los grupos reflejaron diferencias significativas, a favor de los hombres en los factores "autoconcepto físico" y "autoconcepto social". Lo que parece indicar que las mujeres se perciben con autoconcepto más pobre en lo relacionado con su cuerpo y sus relaciones interpersonal.

En síntesis, el análisis de las propiedades psicométricas, ha mostrado que una estructura tetrafactorial es viable y apropiada de acuerdo a los requisitos psicométricos establecidos cuando los informantes son los propias personas. 
La estructura de cuatro factores, atendiendo a criterios estadísticos y sustantivos, ha mostrado adecuados indicadores de ajuste, de fiabilidad y de validez. Sin embargo, consideramos que más estudios son necesarios con el fin de corroborar o refutar los datos obtenidos en la presente investigación.

\section{References:}

1. Abalo, J., Lévy, J., Rial, A. y Varela, J. (2006). Invarianza factorial con muestras múltiples. En J. Lévy (Ed.), Modelización con Estructuras de Covarianzas en Ciencias Sociales (pp. 259-278). Madrid: Netbiblo.

2. Aguirre, J. F., Blanco, H., Peinado, J. E., Mondaca, F. y Rangel, Y. S. (2017). Factorial composition of the Dimensional SelfConcept Questionnaire AUDIM-M in Mexican university students. Revista Electrónica Nova Scientia, 9(18), 627-645.

3. Amezcua, J. A. y Pichardo, M. C. (2000). Diferencias de género en autoconcepto en sujetos adolescentes. Anales de Psicología, 16(2), 207-214.

4. Arbuckle, J. R. (2012). AMOS users guide version 21.0. Chicago, IL: Marketing Department, SPSS Incorporated.

5. Blanco, H., Ornelas, M., Tristán, J. L., Cocca, A., Mayorga-Vega, D., López-Walle, J. y Viciana, J. (2013). Editor for creating and applying computerise surveys. Procedia Social and Behavioral Sciences, 106, 935-940. doi: 10.1016/j.sbspro.2013.12.105

6. Craven, R. G. y Marsh, H. W. (2008). The centrality of the selfconcept construct for psychological wellbeing and unlocking human potential: Implications for child and educational psychologists. Educational and Child Psychology, 25(2), 104-118.

7. Cheung, G. W. y Rensvold, R. B. (2002). Evaluating goodness-offit indexes for testing measurement invariance. Structural Equation Modeling, 9(2), 233-255. doi: 10.1207/s15328007SEM0902_5

8. Fernández-Zabala, A., Goñi, E., Rodríguez-Fernández, A. y Goñi, A. (2015). Un nuevo cuestionario en castellano con escalas de las dimensiones del autoconcepto. Revista Mexicana de Psicología, 32(2), 149-159.

9. Garaigordobil, M., Pérez, J. I. y Mozaz, M. (2008). Self-concept, self-esteem and psychopathological symptoms. Psicothema, 20(1), 114-123.

10. Gelabert, E., García-Esteve, L., Martín-Santos, R., Gutiérrez, F., Torres, A. y Subirà, S. (2011). Psychometric properties of the Spanish version of the Frost Multidimensional Perfectionism Scale in women. Psicothema, 23(1), 133-139. 
11. Goñi, E., Madariaga, J. M., Axpe, I. y Goñi, A. (2011). Structure of the Personal Self-Concept (PSC) Questionnaire. International Journal of Clinical and Health Psychology, 11(3), 509-522.

12. Guillén, F. y Ramírez, M. (2011). Relación entre Autoconcepto y Condición Física en Alumnos del Tercer Ciclo de Primaria. Revista de Psicología del deporte, 20(1), 45-59.

13. Lewis, A. D., Malone, P. S., Huebner, E. S. y Valois, R. F. (2011). Life Satisfaction and Student Engagement in Adolescents. Journal of Youth and Adolescence, 40(3), 249-262. doi: 10.1007/s10964010-9517-6

14. Marsh, H. W. y Martin, A. J. (2011). Academic self-concept and academic achievement: Relations and causal ordering Educational Psicology, 81(1), 59-77. doi: 10.1348/000709910X503501

15. Montero, I. y León, O. (2005). Sistema de clasificación del método en los informes de investigación en Psicología. International Journal of Clinical and Health Psychology, 5, 115-127.

16. Revelle, W. y Zinbarg, R. E. (2009). Coefficients alpha, beta, omega and the glb: comments on Sijtsma. Psychometrika, 74(1), 145-154. doi: 10.1007/s11336-008-9102-z

17. Rodríguez-Fernández, A. y Goñi, A. (2011). La estructura tridimensional del bienestar subjetivo. Anales de Psicología, 27(2), 327-332.

18. Thompson, B. (2004). Exploratory and Confirmatory Factor Analysis. Understanding concepts and applications. . Washington, D C: American Psychological Association.

19. Torres, F., Pompa, E. G., Meza, C., Ancer, L. y González, M. (2010). Relación entre Auto Concepto y Apoyo Social en Estudiantes Universitarios. Daena: International Journal of Good Conscience, 5(2), 298-307.

20. Zhu, J., Wang, X., Liu, Z., Liu, T., Wei, G. y Chen, X. (2016). The Relationship between Self-Concept and Mental Health among Chinese College Students: The Mediating Effect of Social Adjusting. Open Journal of Social Sciences, 4(12), 118-125. doi: 10.4236/jss.2016.412011 